CP, 2015, N6, pp. 81-97. ISSN 2014-6752. Girona (Catalunya). GIL-JUÁREZ, Adriana; VITORES, Anna y FELIU, Joel: Del género a la tecnología y de la tecnología al género: repertorios interpretativos de padres y madres sobre las chicas y los videojuegos. Recibido: 07/07/2015 Aceptado: 16/07/2015

\title{
DEL GÉNERO A LA TECNOLOGÍA Y DE LA TECNOLOGÍA AL GÉNERO: REPERTORIOS INTERPRETATIVOS DE PADRES Y MADRES SOBRE LAS CHICAS Y LOS VIDEOJUEGOS
}

\author{
From gender to technology and from technology to gender: interpretative \\ repertoires of fathers and mothers about girls and video games.
}

\author{
AUTORES: GIL-JUÁREZ, Adriana; VITORES, Anna y FELIU, Joel \\ Profesora Lectora - Universitat Rovira I Virgili - España \\ Investigadora independiente - Reino Unido \\ Profesor Titular - Universitat Autònoma de Barcelona - España \\ adriana.gil@urv.cat; anna.vitores@gmail.com;joel.feliu@uab.cat
}

\section{Resumen}

\begin{abstract}
A través de los discursos obtenidos en distintos grupos de discusión realizados con madres y padres tras un taller de videojuegos en el que participaron con sus hijas e hijos, analizamos las distintas formas de definir y construir el género a la hora de dar sentido a las tecnologías en general, y a los videojuegos en particular. El objetivo de la investigación era observar las relaciones y los discursos de las chicas y el de sus madres o padres respecto a los videojuegos y a la actividad de "videojugar". Recurrimos a los "repertorios interpretativos" como dispositivo analítico con el objetivo de abordar las relaciones entre género y tecnología desde una mirada socio-contextual. El análisis del discurso de los padres y las madres muestra la configuración actual de lo que Valerie Walkerdine (2006) denomina "posición 'femenina' de incompetencia" y cómo opera como posición legitimadora de la "voluntaria" exclusión de las mujeres de lo que se define como tecnológico. Entendemos que las prácticas y los recursos discursivos sobre el género utilizados para dar sentido y definir la relación con los videojuegos y con lo tecnológico, tienen efectos en el uso actual y potencial de las tecnologías por parte de las chicas.
\end{abstract}

\section{Palabras clave}

Género; Videojuegos; Performatividad; Posicionamientos; Repertorios Interpretativos; Brecha digital.

\begin{abstract}
Through the discourses obtained in different discussion groups conducted with mothers and fathers after a workshop with video games in which they participated with their daughters and sons, we analyze the various forms of defining and creating gender to make sense of technologies in general, and video games in particular. The goal of the research was to observe the interaction and talk of girls, their mothers or their fathers about video games and videogaming. We use "interpretive repertoires" as an analytical device in order to address the relationship between gender and technology within a sociocontextual perspective. The analysis of the discourse of fathers and mothers shows the current configuration of what Valerie Walkerdine (2006: 526) called a ''feminine' position of incompetence" and how it operates as a legitimizing position of "voluntary" exclusion of women from what is defined as technological. We understand that practices and discursive resources on gender used to make sense of and define the relationship with video games and technology, have effects on the current and potential use of technology by girls.
\end{abstract}

\section{Key words}

Gender; Video games; Performativity; Positioning; Interpretive Repertoires; Digital divide.

\section{Introducción}

En este artículo exploramos la construcción de género en el discurso de padres y madres a partir de la información obtenida en una investigación destinada a conocer las relaciones entre género y 
tecnología. Específicamente, cuando estas relaciones median en la desafección de muchas chicas hacia los videojuegos y más adelante hacia la tecnología en general.

El objetivo era conocer lo que padres y madres decían de los videojuegos cuando jugaban con ellos con sus hijos e hijas y los discursos que emergían cuando se les preguntaba por su relación con los videojuegos y la tecnología en general. Se suele considerar a las madres como modelos habituales para sus hijas en campos distintos: muestran qué hacen y qué no, en casa, muestran quién se ocupa de quién y de qué y quién es cada uno. En el caso que aquí nos ocupa, nos fijamos en cómo funcionan en tanto que modelos en lo relativo a lo tecnológico. Para analizar sus discursos utilizamos los 'repertorios interpretativos' para fijarnos en la relación género y tecnología de manera socio-contextual, esto es, huyendo de las clásicas explicaciones psicologicistas.

En nuestra discusión argumentamos que los repertorios "generizados" sobre el interés, o la falta de él, por los videojuegos y la tecnología, que emergen en clave tanto genética como evolutiva, son compatibles con los repertorios del interés por los videojuegos como algo personal, reforzando la creencias y los sentimientos de que si a una chica no le gustan las tecnologías tan sólo lo hace en el pleno ejercicio de sus deseos y de su libertad. Esta combinación no sólo tiene efectos vinculados a cómo se enmarcan los videojuegos y las tecnologías desde la posición de lo femenino, sino que refuerzan y fijan las formas hegemónicas de entender qué es y qué no es tecnología y qué es y cómo se es "mujer".

\section{Mujeres y TIC o mujeres en las TIC}

En un artículo publicado en la revista "Gender, Technology and Development”, Wendy Faulkner y Merete Lie sostienen que, en lo relativo a la Brecha Digital de Género, la prospectiva general resulta contradictoria: "optimista en lo que respecta a lo que llamamos mujeres y TIC (esto es, mujeres como usuarias) y pesimista en lo que respecta a las mujeres en las TIC (esto es, las mujeres en el campo de las profesiones TIC)" (Faulkner y Lie, 2007). Su apreciación se asienta en la constatación de que aunque los últimos años se ha igualado el uso TIC entre hombres y mujeres, esto no ha ido acompañado de la incorporación efectiva de mujeres en los sectores estratégicos de la formación, la investigación y la empresa en el ámbito de las TIC, algo que sigue siendo así, o incluso ligeramente peor, en la actualidad (BCS \& eSkills, 2014). Esta distinción entre el ámbito de "mujeres en las TIC" y el de las "las mujeres y las TIC" remite, de hecho a una lógica analítica característica del estudio de la brecha digital de género y, en general, de los estudios feministas sobre la tecnología (Faulkner, 2001; Wajcman, 1991, Gil-Juárez, et al. 2011).

Así, un grueso importante de los trabajos en este ámbito ha estado centrado en estudiar la relación "mujeres y tecnología" esto es, analizar el efecto o la “recepción" de las tecnologías por parte de las mujeres. Durante mucho tiempo fueron estos los trabajos dominantes, puesto que era más habitual encontrar a las mujeres en el lado de la "recepción" de tecnologías y no en el lado de la "producción" de tecnologías. Los estudios clásicos en este ámbito están focalizados en los efectos de las tecnologías en la vida de las mujeres en lo referente al ámbito reproductivo y al del trabajo (el trabajo doméstico y el asalariado). Estos trabajos, tienen en su gran mayoría un marcado tono crítico y pesimista, y coinciden en ver el desarrollo técnico producido a partir de la última parte del siglo XIX como una nueva y perfeccionada forma de dominación masculina. Desde esta óptica se denuncia la exclusión de las mujeres del proceso de creación de tecnologías, no sólo por la falta de control de las mujeres sobre las características del cambio técnico, sino por implicar el desarrollo de técnicas pensadas para las necesidades y los cuerpos de los hombres (Arnold y Faulkner, 1985). Frente a ese pesimismo, es destacable la visión favorable respecto a las nuevas TIC presente en muchas feministas atentas a los efectos de estas tecnologías. En efecto, la visión de las TIC como la promesa de, cuando no una revolución al menos una reforma, no sólo está presente en muchos de "los" teóricos de la sociedad 
informacional (Castells, 1996; Rheingold, 1994) sino también en el llamado "ciberfeminismo" (Reverter, 2001; Sanz 2005, 2008; Wacjam, 2006). Sin embargo, como señala Verónica Sanz, esto no tiene en cuenta la escasez de mujeres participando en el diseño y la producción de contenidos y la experiencia, a menudo negativa, de las mujeres con las tecnologías informáticas con los efectos que conlleva (Sanz, 2005).

En efecto, la escasa participación de las mujeres "en las TIC" nos remite al otro gran bloque de estudios en este campo: la investigación sobre las "mujeres en la tecnología". Sin duda, una de las grandes aportaciones de estos estudios reside en haber visibilizado la persistente escasez de mujeres en este ámbito, analizar la conformación histórica de esta aguda infrarrepresentación femenina en el sector tecnológico y en proponer medidas correctivas de dicha situación. Dentro de este campo, aun cuando existen trabajos referidos a distintos sectores tecnológicos, es el de "las mujeres en las TIC" el que se observa con más atención y preocupación. De hecho, el interrogante que guía la investigación en ese tipo de trabajos es claro y constante: ¿por qué hay tan pocas mujeres en el ámbito de las TIC? Una pregunta que se formula en especial en el caso de los estudios y profesiones en informática ${ }^{2}$, un campo en el que, según señalan numerosos estudios de distintos países, la infrarrepresentación no sólo no se ha corregido sino que se ha agudizado en los últimos años (Anderson et al. 2008; BCS and eSkills, 2014; Boivie, 2010; Ross, 2010; Gal-Ezer, et a. 2009; Glover y Guerrier, 2010; NCWIT, 2010).
La pregunta no es sólo relevante, sino profundamente insidiosa si tenemos presente que este papel minoritario y en declive de las mujeres en la informática corre paralelo a, cuando no viene precedido por, más de 25 años de intervenciones diseñadas no sólo para aumentar el papel de las mujeres en las TIC, sino específicamente para aumentar el número de chicas que acceden a estudios superiores en informática y TICs (Cohoon y Aspray, 2006). Aunque las intervenciones propuestas a lo largo de estos años son variadas, y contienen énfasis distintos en función del marco disciplinar del que se parte, todas ellas parten de una suerte de supuesto común: existen barreras socio-culturales que impiden y/o desincentivan la entrada o el acceso de las mujeres en las TIC (Ahuja, 2002; Cohoon y Aspray, 2006; Gürer y Camp, 2002; Clarke, 2010; Valenduc et al., 2004).

La lógica de las barreras de acceso al sector de las TIC lleva a estudiar las relaciones de las mujeres con esas tecnologías, a investigar cuándo y cómo se produce la segregación respecto a las tecnologías y las prácticas tecnológicas a lo largo de la vida de las mujeres. Nos devuelven, en definitiva, al estudio de "las mujeres y las TIC". Y, entonces, a matizar sustantivamente el optimismo que Faulkner y Lie (2007) pronosticaban en lo que se refiere a las mujeres como usuarias. Así, aunque los estudios coincidan en mostrar que se igualan las ratios de uso (Booth et al.; 2010; Brynin, 2006; Sørensen, 2002), crecen las desigualdades en las habilidades para dominarlas a niveles expertos (Sáinz et al, 2008).

\section{Chicas, TIC y videojuegos}

Las investigaciones preocupadas por promover una relación positiva y de confianza con las TIC destacan que deben tenerse experiencias positivas desde la infancia así como que el aprendizaje debe ser informal. Gran parte de la investigación en este campo ha intentado explicar por qué las niñas no juegan (tanto) a videojuegos como los niños, centrando sus respuestas principalmente alrededor de los contenidos de los videojuegos. Así, se buscan (y se encuentran) en las características de los videojuegos al uso, los más conocidos y vendidos, la explicación del poco interés de las niñas en ellos, destacando tres tipos de contenidos que específicamente disuaden a las niñas: la presencia de violencia (Grodal, 2000; Jansz, 2005; Kafai, 1996; Subrahmanyam y Greenfield, 1998), la construcción de los personajes (masculinos) (Beasley y Standley, 2002; Braun y Giroux 1989; Dill et al, 2005; Escofet y Rubio, 2007; Hartmann y Klimmt, 2006; Ivory, 2006; Janz y Martis, 2007; Provenzo, 1991; 
Scharrer, 2004; Smith et al, 2003; Williams et al. 2009); y, el centrarse en la competición como mecanismo de diversión (Agosto, 2002; Lucas \& Sherry, 2004; Hartmann, y Klimmt, 2006).

Aunque a través de esos estudios se han visibilizado los estereotipos presentes en el grueso de videojuegos, la lógica empleada en ellos corre el riesgo de reificar esos mismos estereotipos en la propia mirada investigadora cuando se considera tanto los videojuegos como el género, dados por hecho, en lugar de preguntarse qué son y cómo llegan a significar (Gil-Juárez, et al. 2011).

\section{Nuestro estudio}

Tal y como dice Torné (s/f), la desafección de las chicas por los videojuegos no se da en el vacío, y no puede desligarse del proceso de subjetivación femenina, de hecho es un signo de que lo femenino se ha incorporado como propio y por ello la desafección va creciendo progresivamente con la edad. Por ello nos interesamos aquí en trabajar las prácticas de videojuego desde un punto de vista discursivo, entendiendo discurso como el conjunto de prácticas que promueven determinadas relaciones sociales (Íñiguez y Antaki, 1994).

Los datos de esta investigación provienen de un trabajo de campo realizado en Barcelona entre abril del 2009 y marzo 2011, que tenía el objetivo de indagar sobre las causas concretas de la desafección de muchas chicas hacia los videojuegos, asumiendo que esta desafección es un primer paso hacia la desafección de las mujeres hacia las tecnologías.

Para la recogida de información se diseñaron y elaboraron seis talleres con videojuegos para madres, padres y sus hijas e hijos ${ }^{3}$. Inmediatamente después de cada taller, se realizaron dos grupos de discusión, uno de padres-madres y otro de chicaschicos. Los talleres, realizados en centros cívicos o ludotecas, fueron de una hora de duración y en el transcurso de los mismos cada pareja (madre/padre con su hija/hijo) podía jugar a cuatro videojuegos, durante quince minutos a cada uno ${ }^{4}$.

Nuestro objetivo al planificar estos talleres era doble: a) por un lado, poder observar directamente el "videojugar" de madres y padres con sus hijos e hijas, prestando especial atención a la performación del género, roles y estereotipos de género, así como a la verbalización de normas y valores; y b) por otro lado, poder obtener información contextualizada sobre las ideas, visiones, afectos y creencias acerca de la tecnología y de los videojuegos por parte de los participantes, es decir, indagar sobre los significados y sentidos de estas creencias y prácticas justo cuando acaban de ser activados de forma práctica en el transcurso de los talleres.

De este modo, las técnicas de recogida de información utilizadas fueron, la observación del juego con videojuegos por parejas en el transcurso de los talleres, y los grupos de discusión de padres y madres por un lado, e hijos e hijas por otro lado, inmediatamente después de los talleres. Tanto el juego de cada pareja como los grupos de discusión fueron registrados con cámara de vídeo.

Los grupos de discusión con las madres/padres (de 5 a 8 personas cada grupo además de un/a investigador/a del equipo) fueron de unos 60-75 minutos de duración y tuvieron lugar inmediatamente a continuación de los talleres. Para la conducción de los grupos se preparó un guion básico de tópicos a abordar que fueron incorporándose al hilo de la conversación surgida. Aquí sólo tratamos los discursos y repertorios que emergieron en los grupos de discusión de los padres-madres, los tópicos a abordar en estos grupos incluían: qué son los videojuegos, cómo se iniciaron con los videojuegos, preferenciasregulación de los videojuegos en el hogar, videojuegos que juegan con sus hijas/os, el juego de sus hijos/as, relaciones con la tecnología en el hogar, relación presente y futura de sus hijas/os con los videojuegos y la tecnología. En la introducción y presentación de los objetivos de la investigación y del grupo de discusión que se hicieron a los participantes, no se mencionó la problemática del género inicialmente, ésta se introducía hacia el final 
de la entrevista si no había aparecido antes de forma espontánea.

Los grupos de discusión fueron transcritos en su totalidad y anonimizados, eliminando datos que pudieran identificar a los participantes. Para la transcripción utilizamos una simplificación del sistema de Jefferson. La traducción del catalán al castellano de algunos extractos (en los grupos de discusión se hablaba catalán y castellano indistintamente) ha sido realizada por el propio equipo investigador.

\section{Procedimiento de análisis}

El análisis de la información obtenida se ha desarrollado a través del análisis del discurso, tomando como referente la forma de comprensión y aproximación al discurso que propone la Psicología discursiva (Davies y Harré, 1990; Potter y Wetherell, 1987; Iñiguez y Antaki, 1994). La adopción de esta perspectiva nos permite adoptar un enfoque analítico con dos características claves. En primer lugar, desarrollar un análisis vinculado y lo más fiel posible a los discursos que se construyen en la vida cotidiana, que tome como punto de partida las interpretaciones que emergen y se generan a partir de la información directa recogida a través de las técnicas mencionadas (la observación y los grupos de discusión). En segundo lugar, se aborda el discurso desde su orientación hacia la acción, examinando aquellas realidades y relaciones sociales mantenidas y promovidas por nuestras prácticas lingüísticas (Gil-Juárez y Vitores, 2011).

Concretamente, recurrimos a la noción de "repertorios interpretativos" entendidos como "las piezas que los hablantes utilizan para construir versiones de acciones, procesos cognitivos y otros fenómenos. Cada repertorio es constituido por una gama restringida de términos usados de una manera específica en lo que respecta a la gramática y al estilo. Estos términos suelen proceder de una o más metáforas clave, y la presencia de un repertorio con frecuencia es delatada por la de ciertos tropos o figuras retóricas" (Potter y Whetherell, 1987: 66). En buena medida, y como señalan Jill Reynolds y Margaret Wetherell (2003) los repertorios consisten en "lo que todo el mundo sabe" sobre un tema, aquello sobre lo que hay una suerte de consenso colectivo tácito tan establecido y familiar que las personas participantes en una conversación son capaces de reconocer la versión del mundo a la que se apela en un repertorio a partir de un pequeño fragmento de la cadena argumentativa y/o cliché del mismo.
En el análisis, nos centramos en identificar los distintos repertorios a los cuales se recurre para dar cuenta de los videojuegos y el género, y de la relación entre ambos, a partir de los enunciados directos recogidos en el transcurso de los grupos de discusión. Así, examinamos la coherencia, coincidencias y divergencias en los conjuntos recursos lingüísticos, construcciones gramaticales y frases hechas utilizadas en la descripción y argumentación durante los grupos de discusión. Al analizar los repertorios se vuelve central atender al contexto de utilización de esos recursos y a su variabilidad: las personas recurrimos a distintos repertorios en una misma conversación, a menudo inconsistentes entre sí, en función de aquello que estemos argumentando, esto es, de acuerdo a las demandas retóricas del contexto inmediato (Wheterell y Potter, 1988). Esta variabilidad permite hacer visibles los dilemas ideológicos presentes en relación a un tema a través del manejo de las inconsistencias que suponen los distintos repertorios presentes para dar cuenta de un tema. Por eso mismo, es importante atender a los efectos distintivos de los repertorios utilizados para comprender cómo participan en la construcción de una determinada forma de entender los videojuegos y su vinculación con el género. Los repertorios interpretativos y los dilemas ideológicos constituyen el andamiaje sobre el que se construyen los hechos en el discurso. Estos aparecen en forma de observaciones -pasa eso, hacen eso, les gusta eso, saben eso-; creencias -yo creo que-; y conocimientos -se sabe que, he leído que, he oído que, he visto que-).

El discurso desde este punto de vista es acción, se está haciendo en el contexto de la acción/actividad a la cual se da respuesta, y todo lo que se dice y hace en ese contexto es el despliegue de respuestas a posibles preguntas y cuestionamientos anticipables. 


\section{Resultados}

En esta práctica discursiva que supusieron los grupos de discusión encontramos:

1) Dilemas ideológicos

El dilema ideológico más importante del que participan los grupos y que resulta vertebrador de los repertorios más recurrentes es el de mujer se nace versus mujer se hace, tal y como lo nombró Simone de Beauvoir (1949) También se hace presente el dilema biológico versus aprendido, como soporte del primero, que además genera un argumento socialmente muy poderoso para justificar y explicar el desinterés y la desafección por la tecnología: los gustos.

2) Repertorios que muestran la configuración del sentido común respecto al género y la tecnología Los tres grandes repertorios que encontramos son: a) el género es un hecho, b) mujer se nace, c) las aficiones son personales.

\section{3) Construcción de hechos}

La consecuencia que tiene la participación discursiva de los grupos en los dilemas mencionados así como el uso que hacen de determinados repertorios es la construcción del hecho que el género nace. No produce el género como construcción social, ni que el género se hace, el género pasa a ser un hecho natural. Pero no siempre emerge el género, cuando no está presente, se reproducen las mismas prácticas de segregación de las chicas y mujeres de la tecnología, sin embargo estas aparecen como producto de decisiones individuales que se distribuyen a lo largo de un eje de género que queda invisibilizado.

El dilema ideológico mujer se nace versus mujer se hace se despliega en una serie de repertorios que encontramos en los discursos sobre tecnología y género. Este dilema tiene como efecto principal reificar la dualidad de género, y mostrar ésta como inevitable. La relación con la tecnología es uno de los ámbitos en que el dilema se muestra en todo su esplendor y no consigue romper con el binarismo de género (algo que por ejemplo la idea de mujer se hace podría conseguir y consigue en otros ámbitos de la cotidianidad).

La existencia de algo como la mujer (universal, ahistórica, homogénea, sin clase social ni marca étnica), se produce en los discursos biologicistas de una forma que nos puede parecer habitual, en tanto que discurso dominante; pero esta mujer universal también se produce en los discursos sobre la socialización en roles (aparentemente más rompedores), al mostrar como ineludible que una persona 'nacida' niña 'inevitablemente' deviene en la mujer por las circunstancias sociopolíticas. En este último lado del dilema esto se logra gracias a la intervención de una figura naturalizada: el individuo. Es la existencia del individuo (natural, universal, ahistórico, con gustos propios e incuestionables), precisamente por su naturaleza individual, quién reproduce la categoría mujer, ya que al quererse hacer mujer, se excluye activamente de las actividades que podrían cuestionar su género, y basa y argumenta estas acciones 'individuales' en sus deseos, gustos $\mathrm{y}$ aficiones personales.

El dilema biológico versus aprendido, en realidad se edifica sobre un consenso implícito: El género es individual, personal y en definitiva respetable $\mathrm{y}$ nadie te puede decir cómo eres o cómo has de ser, o lo que más llama nuestra atención aquí por su relación con los videojuegos: qué te gusta, cuáles son tus gustos. El razonamiento es, si los videojuegos son una afición porque son un juego, nadie puede decirte entonces a qué tienes que jugar, ni si tiene que interesarte o no la tecnología, de hecho no tiene que 'ir contigo si tú no quieres', 'si no te gusta a tecnología, ¿y qué?' 'no se puede obligar a nadie a ir contra sus gustos'.

El razonamiento práctico respecto al género puede justificar y trabajar para naturalizar la exclusión de las mujeres del mundo tecnológico. El razonamiento práctico respecto a los videojuegos puede justificar y trabajar para naturalizar el binarismo esencialista de género.

REPERTORIO 1: EL GÉNERO ES UN HECHO. 
Los videojuegos tienen género en tanto que juegos / las niñas juegan a cosas de niñas/ no hay videojuegos para niñas

A lo largo de las distintas secuencias de conversación el videojuego es a menudo caracterizado como un juego. Los padres y las madres comparan y asimilan los videojuegos a otros juegos que juegan sus hijas e hijos (los coches, las muñecas) o a los que jugaban los mismos padres y madres (el futbolín, las canicas) o a los que todavía juegan (juegos de mesa). En el juego electrónico, de la misma forma que en el juego tradicional, los géneros se ensayan, se ponen a prueba gustos y aficiones y se estabilizan preferencias que coincidan con los mandatos culturales de género.

Mujer 3: si, pero claro, si que...yo ahora que lo pienso, realmente es verdad que...quizás los juegos de la Nintendo y de la Wii, son todos mucho...de fútbol, de conducir

M4: bueno, hay de todo, eh

M3: si, ya, pero los que tienen mis hermanos son estos

M4: ah, claro

M1: (rie)

M3: y quizás ella no se siente atraída

M4: claro

M3: por eso va al ordenador, que en el ordenador pues, puede encontrar otro cosa que...el de cantar, en el ordenador sí, todo esto si que ella lo busca

M2: de Hannah Montana, claro ellas, ellas tienen gustos más diferentes que, que, que los chicos, yo hablo por...

M4: si, si, no

M3: claro, supongo que por eso no se pelean cuando llegan a casa de mis padres, no? el niño y la niña, porque claro, hay un estilo para el niño, y entonces recurre a otro estilo

(Grupo 5)

De este modo, ellos juegan a videojuegos para niños, y ellas juegan a videojuegos para niñas, estableciéndose una manera de construir el género que recrea claramente los estereotipos tradicionales tanto para explicar a qué se juega, cómo para dar cuenta de cómo se juega:
H1 .sí, hay muchas, yo tengo dos niñas y los juegos tienen que ser tranquilitos, quiero decir, les pones juegos de... un juego de matarse y... y no, $y$ no, no les interesan, $y$ en cambio cuando vienen mis sobrinos $y$ ven los juegos que tienen mis hijas, dicen

M2...no les interesan

$H 1$...no les interesan para nada, no hay muertes, claro... es que... ¿es o no es? Eso sí que, el mundo de los niños y de las niñas es muy diferente (Grupo 12)

En este sentido, ser mujer es un hecho y queda claro en el discurso que hay dos mundos diferentes el femenino y el masculino y por lo tanto unas preferencias también diferentes, las femeninas y las masculinas. Las preferencias específicas de las chicas respecto de los videojuegos están claras: no les interesan.

Las expresiones jes o no es? y eso sí expresan que para el locutor nos encontramos ante una verdad indudable, no se puede negociar, es una cuestión de blancos y negros que no admite grises. Un hecho que se adecua al carácter dicotómico de los sexos y de los géneros. En efecto, los hablantes despliegan un conocimiento claro de quién puede y no puede jugar a un videojuego dado, de acuerdo a lo que el mismo videojuego ofrece y de acuerdo a las dos opciones de "ser" (niño o niña) que existen. No puede ser de otro modo.

M4: mi hijo busca jugadores en casa y no encuentra, porque tengo dos niñas y el chaval,

E: aja,

M4: sin embargo, ellas, o sea, él puede jugar a lo de ellas, pero ellas a lo de él

H1: no puede

M3: no puede ser

M4: o sea eso de estar todo el día matando y destruyendo...

M3: no les apetece

H1: yo creo que la play está hecha para....entrecomillas para...niños

(Grupo 1)

Es así que ellas no son jugadoras (o interlocutoras) válidas en el contexto del 
videojuego (se supone que del juego en general, porque niños y niñas no juegan juntos. Las posibilidades no son simétricas en el caso de los videojuegos al contrario de lo que pasa con los juguetes tradicionales, ellos pueden jugar con los de ellas pero ellas no pueden jugar con los de ellos. Siguen siendo dos mundos diferentes caracterizados por distintas preferencias.

H1: pero ves, la Wii es participativa, porque mi mujer es a lo único que podemos...que a veces estamos los tres, oye, cuando no hay nada que hacer, pues vamos a hacer unos bolitos, y nos ponemos los tres alli, pues si empieza a perder el peque ya...gua, gua.ya la tenemos, a ver deja ganar a la mama, le digo, venga a ver que tal, y también tiene que ganar

(Grupo 5)

La madre es alguien que necesita ayuda, compasión, dejarla ganar -como a los pequeñosaunque gane de forma legítima.

Desde este repertorio, ser niña determina apetencias y gustos claros en términos de lo que se puede y no se puede hacer, y esa apreciación es tratada como algo obvio. Sin embargo, las afirmaciones sobre el diseño intencional de los videojuegos, la idea de que quién concibe los videojuegos no piensa en las niñas se presenta como algo discutible, algo a "entrecomillar". Los hablantes tratan esa visión "generizada" del videojuego como una suerte de acusación sexista a una máquina (y a quién la diseña), sin embargo, opera de forma recurrente en las explicaciones de las relaciones de las niñas con los videojuegos. En efecto, nos encontramos de forma reiterada con la explicación de que las niñas juegan menos que los niños a videojuegos, por que no hay muchos videojuegos pensados para que ellas puedan jugar.

M1: es que incluso la PS2, quitando el singstar, que tiene la niña?

M2: no tienen, no tienen

M1: yo lo tengo en casa, y...ya tengo, tiene el singstar, y luego si que tengo los típicos de carreras de coche, para cuando vengan mis sobrinos, engancharlos allí...(rien)
M2: es que una niña es muy raro que se te ponga, a ver, mi hijo lo tiene, a jugar a coches, no, no, y a fútbol

M1. a ver, que hay niñas que también les gustará, pero son las que menos

M2: son las que menos

H1: las que menos

M1: no es muy elevado

(Grupo 5)

Los videojuegos tienen género en tanto que máquinas. El videojuego no es sólo tratado como juego, o no como cualquier tipo de juego. El videojuego es una "máquina". Se construye el videojuego como una máquina tecnológica.

M4: que no le llama tanto la atención, prefiere dibujar, pintar, hacer tiendas en casa, y....jugar a tiendas, cosas más manuales...más normales que...estar con la maquinita, o con la Wii, o con el ordenador (Grupo 7)

H1: pero yo creo una cosa, que ahora, nosotros en la edad esta de las crías que nos ha pillado con los críos, ellos están ahora, vale, tienen máquinas, todos y tal, pero están más con el ordenador el tema del facebook, y cosas de estas, y tiempo que antes estaba, jugando a los sims o cosas de estas, ahora lo dedican $a . .$.

(Grupo 1)

REPERTORIO 2: MUJER SE NACE. Mundos diferentes, preferencias diferentes.

En tanto que tecnología y actividad tecnológica, opera como un contexto paradigmático, en el que el género que lo ocupa se considera una actividad natural. De hecho, se enraíza de tal modo en lo biológico, que resulta fácil entender hasta qué punto desde este marco, una mujer a la que se le dé bien lo tecnológico ve en peligro su feminidad y su heterosexualidad.

H2: yo creo que a los niños les gusta saber, cómo está hecha la...el aparato que están trabajando, con él que están, vale

M3: hace poco se observó que un lóbulo del cerebro, de los hombres, era más mecánico que el 
de las mujeres, entonces se entendía que, por ejemplo, en un examen de conducir, eh... las preguntas que les hacían a los hombres de mecánica y esto, todo perfecto, y a las mujeres ahí las tumbaban, y...bueno, cada uno tenía una actitud, no, pero era un estudio

M3: del cerebro, zonas del cerebro

M2: tú sabes

M3: donde está la lectura, la atención, son diferentes

M1: los hombres mentalmente, cogen mejor toda esta matemática y este cuadro no? de...de la tecnología, y a la mujer nos cuesta mucho más

H2: pero

M1. yo lo estoy estudiando, estoy haciendo electricidad, y lo veo, en mí hay cosas que me las explicas, me las explicas, y...me cuesta, me cuesta, pero es hasta que un día se te enciende la bombilla y lo ves, no? pero cuesta mucho, el automatismo es complicado, para una mujer es complicado, o sea, o te gusta mucho, o no te entra

H1: si, exacto, no te entra

M1: a mí realmente me entran otras cosas más manuales, que no esto, los automatismos, $y, y$ tener que estar ante el ordenador constantemente para programar, o sea, utilizo el autocad para hacer dibujo y tal.... y el autocad me gusta más que no, que no estos otros de automatismos, es como más innato, no? el cerebro no...

(Grupo 1)

Las relaciones que se establecen con lo tecnológico recrean el ordenamiento social en dos géneros y en dos sexos como algo enraizado en la naturaleza. La clásica apelación al cerebro, a los lóbulos del mismo, a lo innato y a lo genético como fundamento de las diferencias en lo que gusta o no gusta fue recurrente en los grupos de discusión cuando se daba cuenta del jugar con tecnologías o trabajar con ellas. Las mujeres no están en lo tecnológico porque su cerebro "es diferente", no se adapta a los requerimientos de lo tecnológico. La diferencia de capacidades en base a argumentos biológicos, se trata como algo que también puede ser discutible (y que es necesario exponer a través de "un estudio" legitimador, una voz externa autorizada), la apelación al cerebro no se maneja en los casos como un argumento si no como un hecho.

La tecnología aparece definida por una serie de características (automatismos, mecánica, abstracción, técnica) habitualmente asociadas a lo masculino y, a su vez, las relaciones que se desarrollan con la tecnología se presentan como "generizadas" de raíz. A las mujeres no les puede dar bien lo tecnológico: es algo que no eligen $\mathrm{y}$ que, por tanto, tiene que costar, si se pretende actuar el género de forma correcta. Tiene que "gustarte mucho" para poder vencer la determinación del género.

Ser mujer implica madurar, las chicas no juegan porque maduran antes, porque son responsables y porque tienen cosas más importantes qué hacer. Así, a través de la relación con los videojuegos se construye la mujer como algo distinto a la niña (un tipo de ser que ya no juega) y las características asociadas a la feminidad-mujer se definen como algo que se adquiere con la maduración, con la edad.

M3: no lo sé, supongo que mira, por la edad, siempre dicen que las niñas son un poco más adelantadas, yo que sé

$H$ : no, eso es verdad, eso yo, habéis madurado antes

M2: en la clase del Alex todas las niñas llevan móvil, y esto en cambio los niños no, y yo tengo una compañera que tiene dos hijos, la niñas, bueno la chica ahora tiene la edad de 18, y el niño ahora tiene 14, y cuando lo tenía más pequeños me lo decían, dice, van a les escolas pia de Terrassa, dice, tuvimos una reunión de estos de cuando se fan de final de curso, no sé cuándo lo hacen sin en la ESO, o no sé qué, y los profesores se lo dijeron, mira, mientras los niños les damos una pelota, y se hinchan de jugar a fútbol, en la playa, las niñas van a ver que tío está más bueno y a la que mejor puesto el bikini. (Grupo 5)

Es por ello que la caracterización del videojuego como juego es también utilizada para explicar por qué, no sólo las niñas, sino sobre todo, las chicas y las mujeres "videojuegan" menos. Es habitual en 
las madres y los padres encontrarse referencias al juego como algo pasado al dar cuenta de porqué sus hijas mayores (en general, a partir de 13 años) no juegan a videojuegos. Nos encontramos aquí la figura de que "las chicas maduran antes que los chicos" que naturaliza también la desafección de las chicas mayores y las mujeres por los videojuegos en tanto que juegos.

Así, la construcción de los videojuegos como un tipo de actividad tecnológica-máquina que habíamos visto, también crea un contexto de construcción del género como fruto de un proceso de evolución. Las chicas, al madurar, al devenir mujeres, estarían interesadas en la parte comunicacional de los ordenadores (las redes sociales) no en los videojuegos en tanto que juegos o en tanto que máquinas.

H2: la mía ahora lo que ha descubierto son las redes sociales de internet

H1: eso también

H2: el facebook...todas estas cosas

H2: y entonces está más tirada al facebook y a la red social que... hablar con las amigas, a....

M2: claro es que ahora, a los 14 años, ya es red social... ya no...

H2: mira que tal....ha empezado a descubrir las redes sociales de internet, facebook, tuenti, todas estas cosas y...

(Grupo 1)

Los chicos también estan en facebook, pero no abandonan el juego para estar en facebook (ya no...). Ser mujer también implica pasar por una determinada socialización.

M2: y a ver, al niño le cortas el pelo de una manera, y a la niña no, la niña quiere el pelo con la pinza bien puesta, y según que ropa ya no se la pone, según que ropa, porque ya empieza...

M3: Es diferente

M2: En cambio los niños van con la camiseta por encima, y van, no de cualquier manera, pero son más desastres

(Grupo 5)

REPERTORIO 3: LAS AFICIONES SON
PERSONALES. Si hay diferencias provienen de diferentes gustos personales y no del género ni de nada social. Quitando el género de la ecuación, la diferencia es entre personas y no entre géneros, pero el efecto es de segregación de las chicas y mujeres.

Los videojuegos, al ser incluidos en las 'Nuevas Tecnologías', dejan de tener género para tener generación. El videojuego es tratado como una nueva tecnología que se conoce y maneja a través de la socialización tecnológica. La categoría de "nativo digital" difumina la del género a la hora de explicar la relación con las nuevas tecnologías. En este contexto, es el acceso y la experiencia con las nuevas tecnologías lo que proporciona las capacidades para manejar las nuevas tecnologías, y los videojuegos definidos ahora en tanto que tales.

M3. yo tengo 3, una niña grande, que tiene 15, que pasó la época gloriosa de los Singstars que venían a cantar todos y a bailar a la casa, y hemos pasado ratos muy chulos, y después tengo el niño este con el que hemos venido ahora, Samuel, a este le encantan los videojuegos, pero muchísimo, y... la pequeña, que coge el móvil de su padre, y no sé cómo lo hace, ya se sabe bajar... los pro... bueno, no sé, es... es extraordinario.

\section{(Grupo 12)}

M4: entonces, claro, a mí me cuesta mucho, me he puesto nerviosa, estos juegos, no entiendo, no sé qué, es como el ordenador, lo utilizas, estás, el facebook, todo, pero no es...claro, has aprendido de grande, $y$ es cómo todo, te cuesta mucho más, nuestras hijas ya...es que mi hija ve un vide, un juego, y dice, a sí, tal, tal, a veces me coge el ordenador, mamá que no, que es...y yo no sé qué ha hecho, y digo, pero qué has hecho, explicamelo, ahora mismo, estábamos jugando, digo, pero niña, pero qué botón tocas, no, mamá, no sé qué, es que han nacido, es que de pequeñas, ya lo tienen todo esto, y están con el ordenador, en casa, siempre, nosotros, como no hemos tenido ordenador hasta grandes, no hemos visto una play, ni una Wii... pues claro, no lo vives así

(Grupo 7) 
Cuando el videojuego es identificado como las nuevas tecnologías, es caracterizado como algo deseable para hijas e hijos, algo que les vuelve competentes en una sociedad llena de nuevas tecnologías.

No es cuestión de capacidad, es cuestión de experiencia. Ellos lo han tenido que hacer. Se aprende a hacerlo haciéndolo. Si tengo que hacerlo lo hago. Si tiene que hacerlo lo hace.

$H$ : yo es un poco igual eh?, no quiere decir que en un momento determinado ella no lo pudiera hacer, pero, yo creo que no ha tenido la necesidad de hacerlo, y aparte, que creo que no le gusta, y se combina con que, quizás a mí me gusta más, tocar la tele, mirar, cositas...típico, compras la tele y ya estás mirando todas las opciones, se puede hacer esto, se puede hacer...un día vino y dice "osti, si se ven dos pantallas en la tele, cómo es esto", y yo, a ella le era igual que se vean dos que se veo una, tú ya está mirando allá que hay una opción que puedes ver, el auxiliar y la...cosas de estas qué...

(Grupo 11)

Las distintas posiciones sociales se traducen en disposiciones personales y la posición social de partida se borra. Las distintas formas de construir el género quedan subsumidas en la visión del género como algo personal. El gusto, no es discutible, ni tiene causa. Es una explicación última.

La distribución social binaria del interés por los videojuegos queda camuflada bajo un consenso que parece atravesar todas las co-construcciones de género-tecnologías: el gusto es un asunto personal. Es una afición, y las aficiones se asumen y conforman desde una perspectiva intimista e individual. Mientras el género de las máquinas o la biología del género pueden discutirse, los gustos y las preferencias, nos sitúan en un terreno distinto, incuestionable.

El gusto y la afectividad por lo tecnológico, se define pues como un escenario central en la actualización y reiteración del sistema de género y de sexo dominante, central por su aparente inocuidad. La apelación al gusto personal borra simbólicamente la conexión que existe entre cualquier práctica social y sistema social del que participa. De este modo, se genera el efecto de generar el sentimiento de que si cada cual adopta las reglas del juego instituidas, tan sólo lo hace en el pleno ejercicio de su propia libertad.

\section{Discusión y conclusiones}

"Pero donde está el peligro, crece también lo que salva" (Hölderlin)

La construcción de hechos que se hace más evidente a través de los dilemas y sus repertorios encontrados, es la del género nace. Y este hecho sirve sobre todo para evitar toda alusión o posible explicación alrededor de que los gustos se aprenden, el género se aprende: y este aprendizaje empieza por lo que se ve en casa. Los repertorios el género es un hecho y mujer se nace, se usan como justificaciones y explicaciones del por qué los padres-madres participantes no han promovido en las chicas el videojuego y el uso de las TIC en general y complementariamente, el repertorio las aficiones son personales reafirma a los padres-madres en su continua actividad de reforzamiento y coconstrucción del género con sus hijas.
El efecto principal de la variabilidad entre los repertorios mujer se nace y las aficiones son personales, es que ambos sirven para eliminar lo social (o al menos ignorarlo) de la ecuación. Se salta de lo biológico a lo individual, sin pasar por lo social.

Sin duda, y como ya señalara Goffman (1977), la identidad de género se sigue considerado en nuestras sociedades una identidad básica $\mathrm{y}$ fundamental: actuamos y entendemos las acciones de otras personas basándonos en estándares de lo que es aceptable en términos del género al que "pertenecemos" según un sistema social marcado por el binarismo femenino-masculino. Esa lógica binaria configura y estabiliza la matriz 
heterosexual (Butler, 1990) que convoca a seguir viendo a la humanidad dividida de forma fundamental en dos categorías básicas y actuar el género en función de esa matriz en distintas situaciones y contextos sociales.

Es por ello que es un 'problema' tener una ubicación ambigua en relación al género. Si la humanidad 'biológicamente' se divide en hombres y mujeres, es un problema no estar bien ubicado en alguna de estas dos categorías, porque entonces todo el sistema 'humanidad' está en cuestión. Por lo tanto es muy importante realizar actividades y acciones que generen 'adecuación' con el propio género. Si a ello le añadimos la creencia de los padres-madres participantes, en la inevitabilidad de la relación entre el género y la tecnología (el hecho de que sea natural, genético, que las mujeres tengan problemas de comprensión de las cosas mecánicas, automáticas o electrónicas), el cóctel de determinismos está servido y es difícil escapar.

No menos importante es la cuestión de los gustos personales, porque es el elemento más importante para poder negar que haya cualquier tipo de discriminación, siempre se puede argumentar que ellas no están interesadas y por eso no están "en las tecnologías". Es el mismo tipo de argumento que usan las jóvenes cuando dicen que gustan de arreglarse y de estar bonitas por el mero placer de hacerlo para ellas mismas, sin que medie ningún tipo de mandato social en ello. Es este argumento, de las aficiones y los gustos como personales, lo que se convierte en una fuente importante de legitimación de las diferencias entre chicos y chicas y sus relaciones con la tecnología.

El ámbito tecnológico se ha ido conformando, simbólica y estructuralmente, como un contexto en el que claramente se da y se actúa esa separación de géneros. Por eso mismo, las situaciones definidas como tecnológicas, se convierten en especialmente centrales para clarificar y reforzar la diferencia de un género respecto al otro. Son situaciones en las que se viven de forma especialmente insidiosa las interpelaciones para definir claramente "qué" eres, "quién" eres. En un momento en el que los discursos sobre la igualdad hacen insostenible la distinción tradicional de géneros en otras situaciones (por ejemplo en relación a las tareas domésticas, las profesiones a ocupar, los deportes que practicar...), los contextos tecnológicos se erigen como espacios que convocan de forma clara a actuar y mantener esa diferenciación. Cuando las tecnologías de la información y la comunicación, convertidas en tecnologías de ocio y tecnologías de relación (Gil, 2007), permean cada una de las esferas de la vida, lo que está en juego no es solamente la cantidad de mujeres que tendremos creándolas y participando de ellas, si no el tipo de sociedad y el sistema de género que tenemos, y el que queremos para nuestra sociedad, si es que queremos alguno. Como reza el verso de Hölderlin que encabeza esta sección, ya utilizado por Heidegger (1954, p.9) al preguntarse por la técnica, si la creación y el consumo de videojuegos en el mundo occidentalizado ayuda a recrear distintos espacios de vida para las personas, en función de la invención y distribución del sistema de género, también puede ayudar a la subversión y problematización de ese mismo sistema.

En, efecto, en los últimos años hemos asistido a la proliferación de un conjunto de trabajos que se centran precisamente en el contexto tecnológico para mostrar la emergencia, marginal pero constante, de formas no heteronormativas de actuar y desplegar la feminidad y la masculinidad (Henwood, 2000; Lagesen, 2008; Dee et al. 2009; Mellstrom, 2009; Varma, 2007; Verges et al. 2009, 2011, 2012) O, mejor dicho, que hacen estallar el binarismo de género en múltiples masculinidades y feminidades precisamente mostrando formas de hacer y decir lo tecnológico y la competencia tecnológica que desbordan el marco de la masculinidad y la feminidad hegemónicas. Al mostrarnos otras formas de decir y hacer lo tecnológico, muestran otras formas de hacer y decir lo femenino y lo masculino.

Como nos decía Foucault (1981, p. 21), pensar, pensar seriamente, nos arrastra indefectiblemente a cambiar de pensamiento. Es el hecho mismo de pensar, el que queda neutralizado cuando se hace 
imposible, o al menos, sumamente costoso, cambiar de pensamiento. En vez de tomar, a niñas y mujeres "videojugadoras", mujeres hackers, o mujeres geekys, como mujeres masculinas o, poco femeninas, deberíamos examinar las formas no

\section{Notas}

1. Este trabajo ha sido posible gracias a la financiación del Ministerio de Educación y Ciencia al proyecto Chicas y videojuegos: procesos de inclusión y exclusión de género en la socialización de las TIC (CSO2008-05203-E).

2. Como muestra de la coincidencia al formular la pregunta objeto de investigación, obsérvense los sucintos títulos de los siguientes trabajos académicos: "Why are There so Few Female Computer Scientists?" (Spertus, 1991) "Why women avoid computer science" (De Palma, 2001); "Why Are So Few Women in Computer Science?" (Beyer et.al, 2002); "Why are there so few women in information technology?" (Rosenbloom et al. 2008); "Technology majors: why are women absent?" (Harris et al. 2009); "Computer Science: Where (and why) have all the women gone?" (Ross, 2010), "Why Aren't More Women in Computer Science?" (Whitecraft y Williams, 2011); Why women and minorities are underrepresented in the computer science major' (Bock, et al., 2013).

3. La puesta en práctica de los talleres se realizó normativas de vivir y hacer el género que nos ofrecen, deberíamos identificar sus rutas invisibles (Gil-Juárez, 2014). Y a su vez, quizás también podamos empezar a pensar, seriamente, qué tecnologías queremos. en colaboración con Marinva, una empresa especializada en el diseño y realización de proyectos educativos y propuestas de comunicación, dinamización y ocio.

4. Seleccionamos cuatro videojuegos para la realización de los talleres, atendiendo a los siguientes criterios: a) que la edad recomendada del juego estuviera comprendida entre los 8 y los 14 años, b) que pudieran intervenir 2 jugadores simultáneamente (una madre o padre y su hija o hijo), c) que se pudiera jugar una partida en 10-15 minutos, d) que hubiera una variedad en la tipología y en los accesorios, y e) incluir juegos de competición y cooperación. Finalmente, y de acuerdo con esos criterios seleccionamos 1 Juego para PC de simulación y cooperación (Spore. Creature creador), 1 Juego con consola creada para jugar en grupo/familia cooperativo (Super Mario Galaxy para Nintendo Wii) 1 Juego con mandos tradicionales de competición deportiva (Shaun White Snowboarding( Playstation) y 1 Juego con accesorios no tradicionales, como la alfombra de baile (Dance Factory para Playstation).

\section{Referencias}

- Agosto, D. E. (2004). Girls and gaming. A summary of the research with implications for practice. Teacher Librarian, 13(3), 8-14.

- Ahuja, M.K. (2002). Women in the information technology profession: a literature review, synthesis, and research agenda. European Journal of Information Systems, 11(1), 20-34.

- Anderson, N., Lankshear, C., Timms, C., \& Courtney, L. (2008). Because it is boring, irrelevant and I don't like computers: Why high school girls avoid professionallyoriented ICT subjects. Computers \& Education, 50(4), 1304-1318.

- Arnold, E., and W. Faulkner. 1985. Smothered by invention: The masculinity of technology. In Smothered by invention: Technology in women's lives, edited by W. Faulkner and E. Arnold, 18-50. London: Pluto.

- $\quad$ BCS \& eSkills (2014). THE WOMEN IN IT SCORECARD 2014 A definitive up to date evidence base for data and commentary on 
women in IT employment and education. http://www.bcs.org/upload/pdf/Women \%20in\%20IT\%20scorecardv2.pdf

- Beasley, B., \& Standley, T. C. (2002). Shirts vs. skins: Clothing as an indicator of gender role stereotyping in video games. Mass Communication and Society, 5(3), 279-293.

- Beyer, S., Rynes, M., Chavez, M., Hay, K.; \& Perrault, J. (2002). Why are so few women in computer science? Comunicación presentada en el Annual Meeting of the American Psychological Association, New Orleans. Extraído el 14 de febrero, 2010 de http://www.eric.ed.gov/PDFS/ED479756. pdf.

- Black, Sue; Jameson, Jean; Komoss, Regine; Meehan, Averil y Numerico, Teresa (2005). Women in Computing: A European and International Perspective. Consultado el 5 de abril del 2010 de la página web del Third European Symposium on Gender and ICT: Working for Change, University of Manchester.

http://ict.open.ac.uk/gender/2005/papers /black.word

- Bock, S. J., Taylor, L. J., Phillips, Z., \& Sun, W. (2013). Women and minorities in computer science majors: Results on barriers from interviews and a survey. Issues in Information Systems, 14(1), 143-152.

- Boivie, I. (2010). Women, Men and programming, a In S. Booth, \& S. Goodman y Gill Kirkup (Eds.), Gender differences in learning and working with technology: Social constructs and cultural contexts (pp. 1-14). Hershey, PA: IGI Global.

- Booth, S.; Goodman, S.; Gill Kirkup (Eds.), Gender differences in learning and working with technology: Social constructs and cultural contexts (pp. 1-14). Hershey, PA: IGI Global.

- Braun, Claude M. J. y Giroux, Josette (1989) Arcade Videogames: Proxemic, Cognitive and Content Analyses. Journal of Leisure Research 21 (2), 92-105.

- Brynin, M. (2006). The Neutered Computer. En R. E. Kraut, M. Brynin \& S. Kiesler (Ed), Computers, phones, and the In- ternet (pp. 84-96). Oxford: Oxford University Press US.

- Butler, J. (1998). Actos performativos y construcción del género. Debate Feminista, 18, 296-314.

- Castells, M. (1997). La era de la información. Volumen I. La sociedad red. Madrid: Alianza Editorial

- Clarke, C. (2010). Computer science. The incredible shrinking woman. In J. T. Misa (Eds.), Gender codes. Why women are leaving computing. Hoboken, NJ: IEEE-CS Press/Wiley.

- Cohoon, J. M. \& Aspray, W. (2006). Women and Information Technology: Research on Underrepresentation. Cambridge: MIT Press.

- Davies, B., \& Harré, R. (1990). Positioning: The discursive production of selves. Journal for the theory of social behaviour, 20(1), 4363.

- De Palma, P. (2001). Viewpoint: Why women avoid computer science. Communications of the ACM, 44 (6), 27-30.

- Dee, H. M., Petrie, K. E., Boyle, R. D., y Pau, R. (2009). Why are we still here?: experiences of successful women in computing. SIGCSE Bulletin 41 (3), 233-237.

- Dill, K. E., Gentile, D. A., Richter, W. A., \& Dill, J. C. (2005). Violence, sex, race and age in popular video games: A content analysis. In E. Cole \& J. Henderson Daniel (Eds.), Featuring females: Feminist analyses of the media (pp. 115-130). Washington, DC: American Psychological Association.

- Escofet Roig, A., \& Rubio Hurtado, M. J. (2007). "La brecha digital: género y juegos de ordenador. Revista Iberoamericana sobre Calidad, Eficacia and Cambio en la Educación 5(1), 63-77.

- Faulkner, W. (2001). The technology question in feminism: A view from feminist technology studies. Women's Studies International Forum, 24, 1, 79-95. doi:10.1016/S0277-5395(00)00166-7.

- Faulkner, W., \& Lie, M. (2007). Gender in the Information Society: Strategies of inclusion. Gender, Technology and Development, 
11, núm. 2, 157-177. doi: $10.1177 / 097185240701100202$.

- Gal-Ezer, J.; Shahak, D. \& Zur, E. (2009). Computer science issues in high school: gender and more... SIGCSE Bulletin, 41(3), 278-282.

- Gil, A. y Vall-llovera, M. (Coords.) (2006). Jóvenes en Cibercafés: la dimensión física del futuro virtual. Barcelona: Editorial UOC.

- Gil, A. (2007). Nuevas Tecnologías de Relación. En J. Romay (Ed.), Perspectivas y Retrospectivas de la Psicología Social en los Albores del Siglo XXI. Madrid: Editorial Biblioteca Nueva.

- Gil-Juarez, A.; Feliu, J. y Vitores, A. (2010). Performatividad Tecnológica de Género: Explorando la Brecha Digital en el mundo del Videojuego. Quaderns de Psicologia, 12 (2), 209-226. Disponible en http: / / www.quadernsdepsicologia.cat/articl e/view/758

- Gil-Juárez, A. y Vitores, A. (2011). Comunicación y Discurso. Barcelona: Editorial UOC.

- Gil-Juarez, A., Vitores, A., Feliu, J. y Vallllovera, M. (2011). Brecha digital de género: Una revisión y una propuesta. En Barrios Vicente, I. M. (Coord.) Mujeres y la sociedad de la Información. Revista Teoría de la Educación: Educación y Cultura en la Sociedad de la Información. Vol. 12, no 2. Universidad de Salamanca, pp. 25-53. Disponible en http: / / campus.usal.es/ revistas trabajo/in dex.php/revistatesi/article/view/8272/827 $\underline{6}$

- Gil-Juárez, A. (2014). Trayectorias de vida tecnológica y género: factores psicosociales implicados en el acceso a las titulaciones de ingeniería informática. Instituto de la Mujer. Disponible

en http: / / www.inmujer.gob.es/areasTematicas /estudios/estudioslinea2014/docs/Trayecto rias vida tecnologica genero.pdf

- Glover, J., \& Guerrier, Y. (2010). Women in hybrid roles in IT employment: A return to 'nimble fingers'? Journal of Technology Management \& Innovation, 5(1).
doi:10.4067/S0718-27242010000100007.

- Goffman, E. (1977). The arrangement between the sexes. Theory and Society, 4, 301331.

- Grodal, T. (2000). Video games and the pleasure of control. In D. Zillman \& P. Vorderer (Eds.), Media entertainment: The psychology of its appeal (pp. 197-213). Mahwah, NJ: Lawrence Erlbaum.

- Gürer, D., \& Camp, T. (2002). An ACM-W literature review on women in computing. SIGSCE Bulletin, 34(2), 121-127.

- Hartmann, T., \& Klimmt, C. (2006). Gender and computer games: Exploring females' dislikes. Journal of Computer-Mediated Communication, 11(4), 910-931.

- Harris, N., Cushman, P., Kruck, S. E. \& Anderson, R. D. (2009). Technology Majors: Why Are Women Absent? Journal of Computer Information Systems, 50 (2), 2330.

- Henwood, F. (1998). Engineering difference: Discourses on gender, sexuality and work in acollege of technology. Gender \& Education, $\quad 10, \quad 1, \quad 35-49$. doi:10.1080/09540259821087.

- Íñiguez, L.; Antaki, L. (1994). El análisis del discurso en Psicología Social. Boletín de Psicología Social, (44), 57-75.

- Ivory, J. D. (2006). Still a man's game: Gender representation in online reviews of video games. Mass Communication and Society, 9(1), 103-114.

- Jansz, J. (2005). The emotional appeal of violent video games for adolescent males. Communication Theory, 15(3), 219-241.

- Jansz, J. \& Martis, R. G. (2007). The Lara phenomenon: powerful female characters in video games. Sex Roles, 56: 141-148.

- Kafai, Y. B. (1996). Electronic playworlds: Gender differences in children's constructions of video games. In Y. B. Kafai \& M. Resnick (Eds.), Contructionism in practice: Designing, learning, and thinking in a digital world , (pp. 97-123). Mahwah, NJ: Lawrence Erlbaum Associates.

- Kafai, Y. B. (1998). Video game designs by girls and boys: Variability and consistency of 
gender differences. En Justine Cassell y Henry Jenkins (Eds.), From Barbie to Mortal Kombat gender and computer games (pp. 90-117). Cambridge: MIT Press.

- Lagesen, V. A. (2008). A Cyberfeminist utopia?: Perceptions of gender and computer science among Malaysian women computer science students and faculty. Science, Technology \& Human Values, 33, 5-27. doi:10.1177/0162243907306192.

- Lucas, K., \& Sherry, J. L. (2004). Sex differences in video game play: A communicationbased explanation. Communication Research, 35(5), 499-523.

- Mellström, U. (2009). The intersection of gender, race and cultural boundaries, or why is computer science in Malaysia dominated by women? Social Studies of Science, 39, 885-907. doi:10.1177/0306312709334636.

- National Center for Women \& Information Technology (NCWIT) (2010). NCWIT Scorecard: A Report on the Status of Women in Information Technology. Extraído el 15 de marzo, 2010, de http://ncwit.org/pdf/NCWITScorecard201 0 Workforce.ppt.

- Platman, K., \& Taylor, P. (2004). Workforce ageing in the new economy: a comparative study of information technology employment. University of Cambridge

- Potter, J., \& Wetherell, M. (1987). Discourse and social psychology: Beyond attitudes and behaviour. Sage

- Provenzo, E. F. (1991). Video kids: Making sense of Nintendo. Cambridge, MA: Harvard University Press.

- Reverter, S. (2001). Reflexiones en torno al Ciberfeminismo. Asparkia. Investigació Feminista, 12, 35-52

- Reynolds, J., \& Wetherell, M. (2003). The discursive climate of singleness: The consequences for women's negotiation of a single identity. Feminism \& Psychology, 13(4), 489-510

- Rheingold, H. (1994). Realidad virtual. Gedisa.

- Rosenbloom, J. L., Ash, R. A., Dupont, B., \& Coder, L. (2008). Why are there so few women in information technology? Assessing the role of personality in career choices. Journal of Economic Psychology, 29(4), 543-554. doi:10.1016/j.joep.2007.09.005.

- Ross, L. R. (2010). Computer science: Where (and why) have all the women gone? Retrieved from http://auspace.athabascau.ca:8080/dspace/ bitstream/2149/2551/1/Lynda\%20Ross_pape r.rtf [30 marzo del 2011].

- Sáinz, M., Castaño, C. \& Artal, M. (2008). Review of the concept "digital literacy" and its implications on the study of the gender digital divide. Working paper series WP08001. Extraído el 28 de marzo, 2011, de http://www.uoc.edu/in3/dt/eng/sainz_cas tano artal.pdf.

- Sanz, V. (2005). Women's careers in Computer Engineering. Case study: Technical University of Madrid. Consultado el 3 de abril del 2010 de la página del Third European Symposium on Gender and ICT: http://ict.open.ac.uk/gender/2005/papers Isanz .ppt

- Sanz, V. (2008). Mujeres e Ingeniería Informática: el caso de la Facultad de Informática de la UPM. Arbor, 184 (733), 905-915.

- Scharrer, E. (2004). Virtual violence: Gender and aggression in video game advertisements. Mass Communication and Society, 7(4), 393-412.

- Smith, Stacy L. ; Lachlan, Ken y Tamborini, Ron (2003). Popular video games: Quantifying the presentation of violence and its content. Journal of Broadcasting and Electronic Media, 47 (1), 58- 76.

- Sørensen, K. H. (2002). Love, Duty and the S-curve: An Overview of Some Current Literature on Gender and $\mathrm{ICT}^{\text {ele }}$. En K. H. Sørensen \& J. Stewart (Ed), Digital Divides and Inclusion Measures. A Review of Literature and Statistical Trends on Gender and ICT, STS Report 59 (pp. 1-36). Trondheim: NTNU, Centre for Technology and Society.

- Spertus, E. (1991). Why are there so few female computer scientists?. MIT Artificial 
Intelligence Laboratory Technical Report number 1315. Retrieved from: ftp://publications.ai.mit.edu/aipublications/pdf/AITR-1315.pdf.

- Subrahmanyam, K.y Greenfield, P. M. (1998). Computer games for girls: what makes them play. En J. Cassell y H. Jenkins (Eds.) From Barbie to Mortal Kombat: Gender and Computer Games (pp. 46-71). Cambridge: MIT Press.

- Torné, M. (s/f). Videojocs i subjectivitat femenina. Construcció de la desafecció. Tesis Doctoral. Universitat Autònoma de Barcelona.

- Valenduc, G., Vendramin, P., Guffens, C., Ponzellini, A. M., Lebano, A., D'Ouville, L., Collet, I., Wagner, I., Birbaumer, A., Tolar, M. \& Webster, J. (2004). Widening Women's Work in Information and Communication Technologies. European Commission (IST-2001-34520). Namur: Fondation Travail-Université.

- Varma, R. (2007). Decoding the female exodus from computing education. Information, Communication and Society, 10(2), 179-191. doi:10.1080/13691180701307396.

- Vergés, N., Cruells, E., \& Hahce, A. (2009). Retos y potencialidades para las mujeres en la participación del desarrollo de la sociedad de la información. Feminismo/s. 14, 163-182. Retrieved from: http://www.donestech.net/files/feminismo s14donestech.docVon

- Vergés, N. (2011). Gender and ICT: The selfinclusion process of women in ICT. An approach from artistic technologists and computer technolo- gists. PhD Thesis. Universitat Oberta de Catalunya.

- Vergés, N. (2012). De la exclusión a la autoinclusión de las mujeres en las TIC. Motivaciones, posibilitadores y mecanismos de auto-inclusión. Athenea Digital. Revista de Pensamiento e Investigación Social,12(3), 129. 150.

- Wajcman, J. (1991). Feminism confronts technology. Cambridge: Polity Press.

- Walkerdine, V. (2006). Playing the game: Young girls performing femininity in video game play. Feminist Media Studies, 6(4), 519 537.

- Wajcman, J.(2006). El Tecnofeminismo. Madrid: Cátedra.

- Wetherell, M.; Potter, J. (1988). Discourse analysis and the identification of interpretative repertoires. (pp. 168-183). En C. Antaki (Ed). Analysing everyday explanation: A casebook of methods. Thousand Oaks (CA): Sage.

- Whitecraft, M. A., \& Williams, W. M. (2010). Why aren't more women in computer science? In A. Oram \& G. Wilson (Eds.), Making software (pp. 221-238). Sebastopol: O'Reilly Media, Inc.

- Williams, D., Martins, N., Consalvo, M., \& Ivory, J. D. (2009). The virtual census: Representations of gender, race and age in video games. New Media and Society, 11(5), 815834.

\section{Forma de Citación}

GIL-JUÁREZ, Adriana; VITORES, Anna y FELIU, Joel: Del género a la tecnología y de la tecnología al género: repertorios interpretativos de padres y madres sobre las chicas y los videojuegos. Revista Communication Papers, No 6 , páginas 81 a 97. Departamento de Filología y Comunicación de la Universidad de Girona. Recuperado el _ de de 2 de: http: / / www.communicationpapers.es 